A New Type of LD 3 havel ength Pul sed Laser for PDD and PDT

\begin{tabular}{|l|l|}
\hline $\begin{array}{l}\text { j our nal or } \\
\text { publ i cat i on t i t l e }\end{array}$ & Pr oceedi ngs i n 13 I PA \\
\hline page range & $121-125$ \\
\hline year & $2011-05$ \\
\hline URL & ht t p: //hdl . handl e. net /10098/7424 \\
\hline
\end{tabular}




\title{
A New Type of LD 3 Wavelength Pulsed Laser for PDD and PDT
}

\author{
Norio Miyoshi $^{1}{ }^{*}$, Kyo Kume ${ }^{2}$, Kotaro Tsutumi $^{3}$, Yukihiro Fukunaga ${ }^{1}$, Shinnji Ito ${ }^{1}$, \\ Yoshiaki Imamura ${ }^{4}$, and Andriana B. Bibin ${ }^{1}$ \\ ${ }^{1}$ Division of Tumor Pathology, Department of Pathological Medicine, Faculty of Medical Sciences, and \\ ${ }^{4}$ Department of Clinical Pathology, Attached Hospital, National University of Fukui, \#23-3, Matsuoka, \\ Eiheiji-cho, Yoshida-gun, Fukui 910-1193, Japan, \\ 2Wakasa-Wan Energy Research Center. \#64-52-1, Nagatani, Tsuruga-city, Fukui, 914-0192, Japan, \\ 3 Yamaki Ltd. Co., \#2-31-18, Minami-Tsukushino,Machida-city, Tokyo, 194-0002, Japan. \\ * Email: corresponding author: nmiyoshi@u-fukui.ac.jp
}

We developed an excitation laser and the spectrophotometer for the fluorescent diagnosis of tumor in the case of 5-ALA as shown in Figure 4. In actual, the removed tissue of glioblastoma was emitted the red fluorescence image shown in Figure 1 of Pp-IX $4 \mathrm{hr}$ after the oral administration of 5-ALA aqueous solution before the operation. Especially, in the labeled position of 1 area in the white light image (left upper image) of the GBM is emitted red as shown in the fluorescence image (right bottom image) will be fallen behind to remove in white light on the surgical operation.

Figure 1.

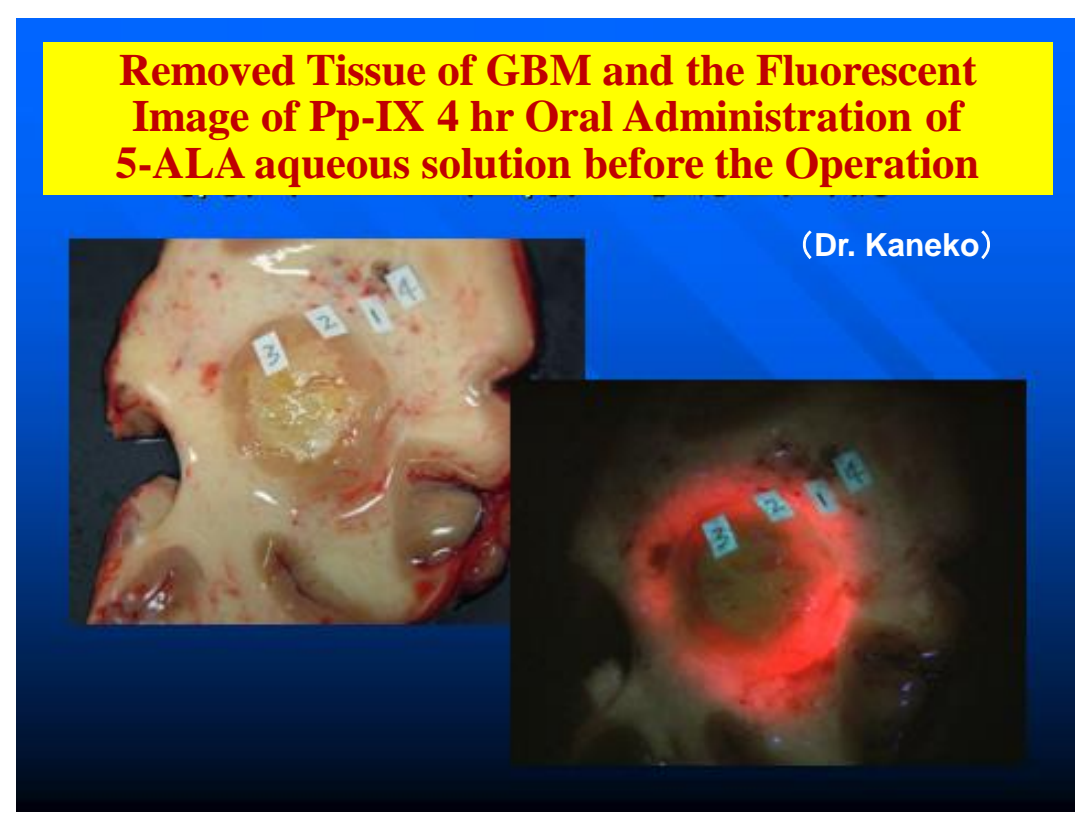

On the other hand, we take to move on the PDT studies. The excitation spectrum of $\mathrm{Pp}$-IX were changed from pattern A to B during the irradiation at $635 \mathrm{~nm}$ for $40 \mathrm{~J}$ in the HL-60 cells suspension incorporated 5-ALA as shown in Figure 7. We observed the changing spectra (from A to B) of Pp-IX photoproduct during photo-irradiation At the 
wavelength range more than $600 \mathrm{~nm}$, the pair of fluorescence excitation (ex) and emission (em) spectra of A (Pp-IX) and B (photoproduct of Pp-IX) were normalized at the each peaks as shown in Figure 2. In actual, the excitation peak at $630 \mathrm{~nm}$ was decreasing with increasing the excitation peak at $665 \mathrm{~nm}$ during the photo-irradiation. As it has been reported about the photoproducts of Pp-IX by many researchers, chlorine derivative by Moan group $(1,2,3)$, we can also estimate that it will be a chlorine-like derivative from the matting with the excitation $(665 \mathrm{~nm})$ and emission $(670 \mathrm{~nm})$ peaks of the derivative. Furthermore, the product plays photoactive as a photosensitizer as following figures. On the other hand, it was reported that the photoproduct will a hydroxyl aldehyde derivative of Pp-IX reported by Dietel, et al. (4) as the product of Pp-IX.

\section{Figure 2.}

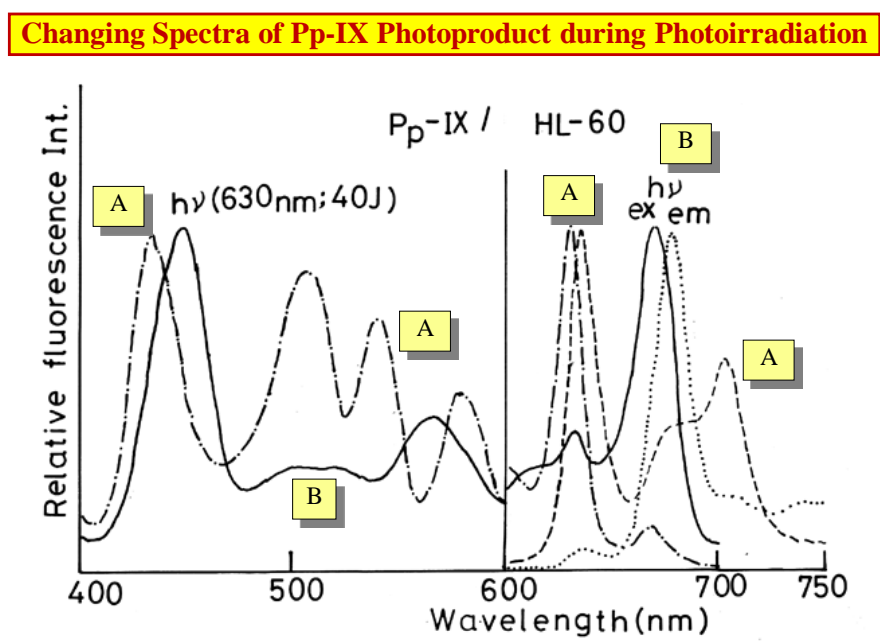

We developed a dual-color $(635,670 \mathrm{~nm}$ ) diode laser system (DLD-R2) produced from SMI-ALApromo Ltd. Co. (Tokyo) in Figure 8. Furthermore, we developed a new disk pulsed laser for three wavelengths at 659,664 , and $669 \mathrm{~nm}$ as shown in Figure 9. These laser will be used for the photoproduct of Pp-IX. We has developed a LD pulsed double (635 and $665 \mathrm{~nm}$ ) laser made by hand as shown in Figure 10. On the CW laser, the longer wavelength laser beam enhanced the PDT effect of tumor in the case of shorter wavelength irradiation at $635 \mathrm{~nm}$ only. 
Figure 3.

\section{LD Pulsed Double Laser (Hand made, 635+665 nm)}

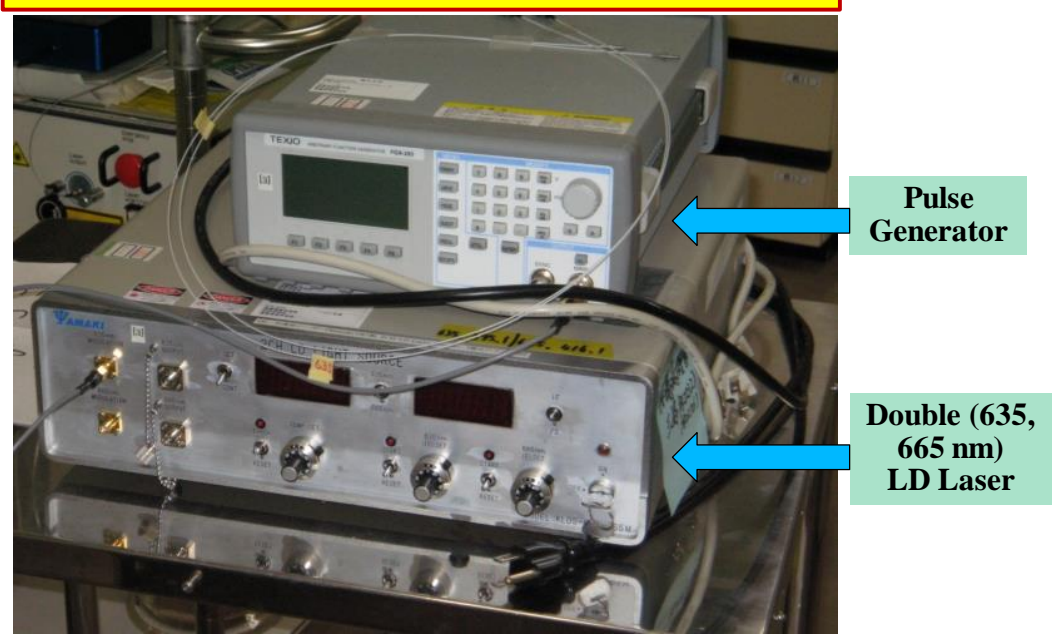

Figure 4.

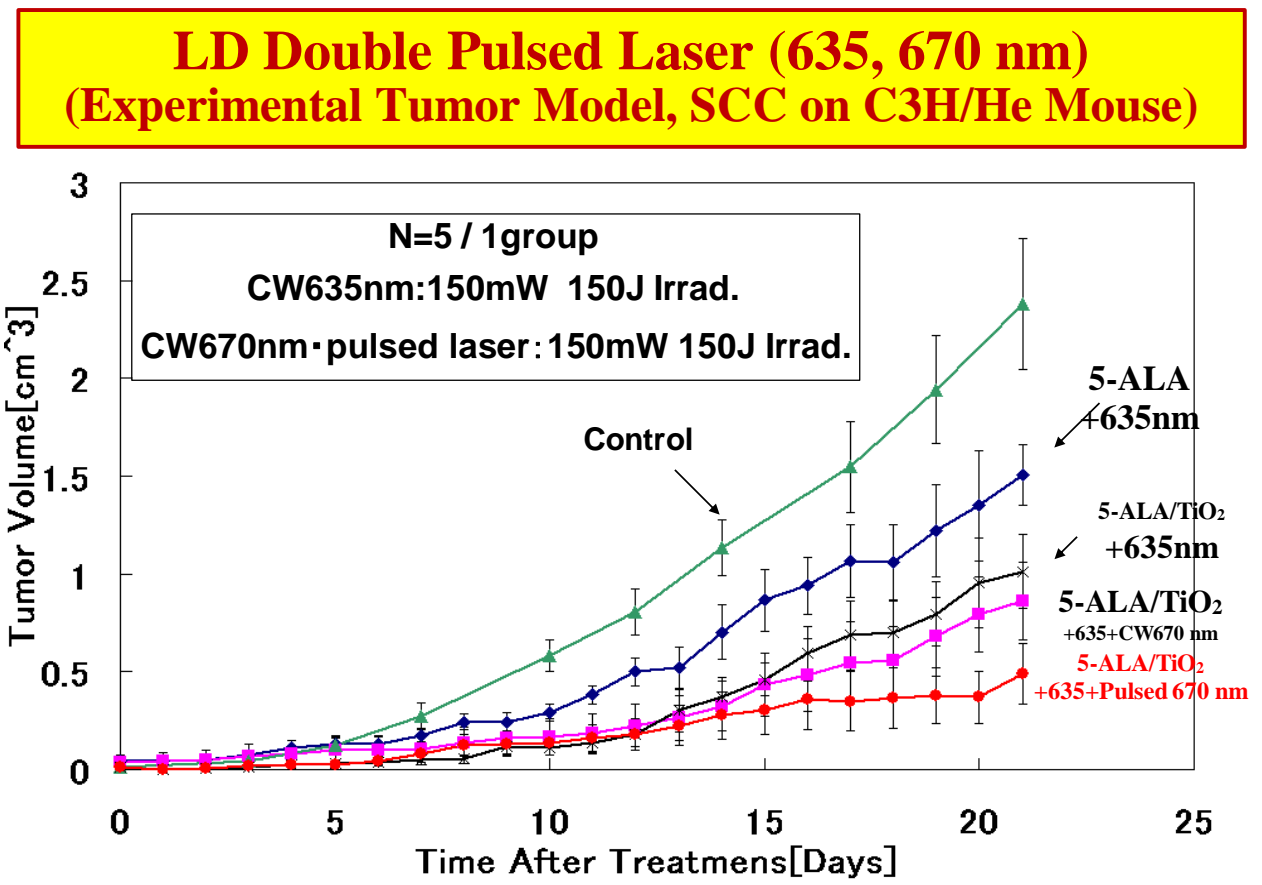

In the case of using the $\mathrm{TiO}_{2}$ particles, we have applied the ultrasound irradiation 
treatments for the experimental tumor model combined with PDT by the instruments (G:generator, A:amplifier, C:cooling water, and $\mathrm{P}$ :ultrasound probe) as shown in Figure 13. As we expected the cavitations effect by ultrasound against the particles, we irradiated the ultrasound $(1 \mathrm{MHz})$ for 10 min combined with PDT as shown in Figure 13. It was identified that the enhancing antitumor effects by ultrasound and PDT-combined $\mathrm{TiO}_{2}$ particles, see Figure 5.

Figure 5.

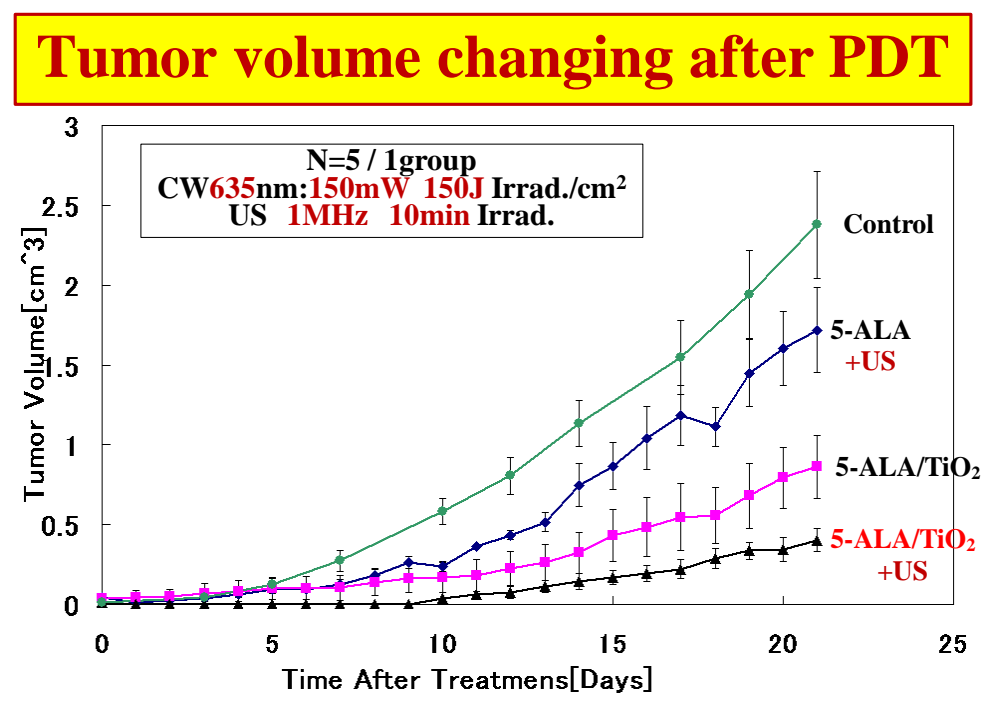

In future, we are continuing to apply the other combination therapy with PDT against the animal tumor models.

\section{ACKNOWLEDGMENTS}

We would like to thank for Mr. H. Katoh for his help in H.\&E. staining of many tumor samples. We would also thank the grant-in-aid to the collaboration research of Wakasa-wan Energy Research Center, Monbu-Kagaku-shou (Kiban-Kenkyu-B), and the Front Analytical Technology and Instrument Development Project Grant-in-Aid by Japanese Science and Technology Promotion System (JST).

\section{References}

(1) Baqdonas S, Ma LW, Lari V, Rotomskis R, Juzeras P, Moan J. Phototransformations of 5-aminolevulinic acid-induced protoporphyrin IX in vitro: a spectroscopic study. Photochem. Photobiol., 72 (2): 186-192 (2000).

(2) Ma L, Baqdonas S, Moan J. The photosensitizing effect of the photoproduct of protoporphyrin IX. J. Photochem. Photobiol. B, 60 (2-3): 108-1013 (2001).

(3) Juzenas P, Lani Y, Baqdonas S, Rotomskis R, Moan J. Fluorescence spectroscopy of normal mouse skin exposed to 5-aminolaevulinic acid and red light. J. Photochem. Photobiol. B, 61 (1-2): 78-86 (2001).

(4) Dietel W, Fritsch C, Pottier RH, Werndenburg R. 5-aminolaevulinic-acid-induced formation of different porphyrins and their photomodifications. Lasers Med. Sci., 12 (3): 226-236 (1997). 